\title{
A REMARK ON A COMPARISON THEOREM OF SWANSON
}

\section{KURT KREITH}

In [1] C. A. Swanson proves a comparison theorem for sufficiently regular, second order elliptic equations of the form

$$
\begin{gathered}
L^{*} u \equiv \sum_{i, j=1}^{n} D_{i}\left(a_{i j}^{*} D_{j} u\right)+2 \sum_{i} b_{i}^{*} D_{i} u+c^{*} u=0, \\
L v \equiv \sum_{i, j=1}^{n} D_{i}\left(a_{i j} D_{j} v\right)+2 \sum_{i} b_{i} D_{i} v+c v=0,
\end{gathered}
$$

defined in a domain $R$ with piecewise continuous unit normal on the boundary $B$. Given that $L$ is a strict Sturmian majorant of $L^{*}$ and that there exists a nontrivial solution of (1) satisfying $u=0$ on $B$, Swanson shows that every solution of (2) has a zero in $\bar{R}$. This result is not "strong" in the sense of [2] where it is shown that under similar hypotheses in the selfadjoint case, every solution of (2) must vanish in the interior of $R$.

The purpose of this note is to point out that if $B$ is of bounded curvature, then one can use the method of [1] to arrive at this stronger conclusion even in the nonselfadjoint case. Specifically, if it can be shown that every solution of (2) which is not zero in $R$ and vanishes at a point $\mathbf{x}_{0} \in B$ must satisfy $(\partial v / \partial v)\left(\mathbf{x}_{0}\right) \neq 0$, then it is clear from the proof that the Lemma of [1] can be altered to read: "Suppose $g$ satisfies $g \operatorname{det}\left(a_{i j}\right)>-\sum_{i=1}^{n} b_{i} B_{i}$. If there exists $u \in \mathcal{D}$ not identically zero such that $J[u] \leqq 0$, then every solution $v$ of $L v=0$ vanishes at some point of $R$." A strong version of Swanson's comparison theorem follows readily from this change, and in the case of ordinary differential equations (i.e. $n=1$ ) this fact is observed in [1].

If $c \leqq 0$ near $B$ and $B$ is of bounded curvature, then it follows from the Hopf maximum principle [3] that $(\partial v / \partial \nu)\left(x_{0}\right) \neq 0$ whenever $v\left(x_{0}\right)=0, x_{0} \in B$. However, even if $c$ is merely bounded, the same conclusion can be derived.

To see this we assume $v<0$ in $R$ and $v\left(x_{0}\right)=0$ for some $\mathrm{x}_{0} \in B$. Without loss of generality we may assume that $B$ is tangent to the plane $x_{1}=b$ and that the exterior normal to $B$ at $x_{0}$ is in the positive $x_{1}$ direction. It is known (see [4, p. 73]) that for $(b-a)$ sufficiently small there exist positive constants $\alpha, \beta$ such that

Received by the editors November 2, 1967. 


$$
\begin{aligned}
w(\mathrm{x}) \equiv 1-\beta e^{\alpha\left(x_{1}-a\right)}>0 & \text { for } a \leqq x_{1} \leqq b ; \\
L w \leqq 0 & \text { for } a \leqq x_{1} \leqq b .
\end{aligned}
$$

Furthermore a direct computation (see [4, p. 72]) shows that the Hopf maximum principle applies to $v / w$ in the intersection of the slab $a<x_{1}<b$ with $R$. Since $v / w$ has a nonnegative maximum at $\mathrm{x}_{0}$, it follows that at $x_{0}$

$$
0<\frac{\partial}{\partial \nu}\left(\frac{v}{w}\right)=\frac{w\left(\partial v / \partial x_{1}\right)-v\left(\partial w / \partial x_{1}\right)}{w^{2}}=\frac{1}{w} \frac{\partial v}{\partial \nu} .
$$

Therefore $\partial v / \partial \nu>0$ at $\mathbf{x}_{0}$ and the strong comparison theorem follows.

These remarks can also be used to strengthen some of the conclusions of [5] for comparison theorems in unbounded domains.

\section{BibliograPHY}

1. C. A. Swanson, A comparison theorem for elliptic differential equations, Proc. Amer. Math. Soc. 17 (1966), 611-616.

2. K. Kreith, $A$ strong comparison theorem for selfadjoint elliptic equations, Proc. Amer. Math. Soc. 19 (1968), 989-990.

3. E. Hopf, $A$ remark on linear elliptic differential equations of the second order, Proc. Amer. Math. Soc. 3 (1952), 791-793.

4. M. H. Protter and H. F. Weinberger, Maximum principles in differential equations, Prentice-Hall, Englewood Cliffs, N. J., 1967.

5. C. A. Swanson, Comparison theorems for elliptic equations on unbounded domains, Trans. Amer. Math. Soc. 126 (1967), 278-285.

University of California, Davis 